\title{
Abdome agudo obstrutivo por volvo cecal: relato de caso
}

\author{
Acute obstructive abdomen by ceacal volvulus: case report \\ Letícia Valerim dos Santos ${ }^{1}$, Vitória Lana Massarente ${ }^{1}$, Laura Beatriz Pires Almeida Leber ${ }^{1}$, \\ Stephanie Corradini Tormin ${ }^{1}$, Karen Falcão Britto ${ }^{2}$, Paulo de Azeredo Passos Candeláriaa ${ }^{3}$
}

\begin{abstract}
Resumo
O volvo de ceco é responsável por aproximadamente 1\% dos casos de obstruções intestinais agudas. Ele ocorre devido a torção dessa estrutura em torno do seu próprio mesentério. O quadro clínico mais comum apresentado é dor abdominal aguda, associada a distensão, vômitos, náuseas, hipertimpanismo com ou sem presença de massa palpável. O diagnóstico pode ser confirmado através de radiografia simples abdominal, evidenciando dilatações de alças intestinais e ausência de gás no ceco; também é possível utilizar exames de imagem como o enema opaco e a tomografia computadorizada de abdome, que indicam a localização da torção com maior precisão. As principais complicações desses casos são obstrução intestinal, isquemia e necrose, podendo levar a perfuração e morte, dessa forma, opta-se, na maioria dos casos, por uma laparotomia exploradora de forma emergencial. Na conduta, podemos optar por desvolvulação com cecopexia ou até mesmo hemicolectomia direita.
\end{abstract}

Descritores: Volvo intestinal, Doenças do ceco, Obstrução intestinal

\begin{abstract}
Ceacal volvulus describes torsion of the caecum around its mesentery which often results in obstruction. It accounts for approximately $1 \%$ of acute bowel obstruction cases. The most common clinical presentation is acute abdominal pain, associated with distension, vomiting, nausea, hyperthympanism with or without presence of palpable mass.
\end{abstract}

1. Acadêmica da Faculdade de Ciências Médicas da Santa Casa de São Paulo - $6^{\circ}$ Ano do Curso de Graduação em Medicina

2. Médica Residente da Irmandade da Santa da Casa de Misericórdia de São Paulo-Departamento de Cirurgia

3. Professor Instrutor da Faculdade de Ciências Médicas da Santa Casa de São Paulo - Departamento de Cirurgia

Trabalho realizado: Faculdade de Ciências Médicas da Santa Casa de São Paulo. Departamento de Cirurgia / Irmandade da Santa Casa de Misericórdia de São Paulo. Departamento de Cirurgia Endereço para correspondência: Vitória Lana Massarente. Rua Martinico Prado,128 - apto 64 - Vila Buarque - 01224010 - São Paulo-SP-Brasil.E-mail:vi_lana_27@hotmail.com
The diagnosis can be confirmed by abdominal radiographs, showing distension of a loop of large bowel and absence of gas in the caecum; it is also possible to use barium enema and abdominal computed tomography, which indicate the location of the torsion with greater precision. The main complications of these cases are intestinal obstruction, ischemia and necrosis, which can lead to perforation and death, in this way, it is opted in most cases to perform an exploratory laparotomy. The treatment can include reuction with cecopexy or even right hemicolectomy.

Keywords: Intestinal volvulus, Cecal diseases, Intestinal obstruction

\section{Introdução}

Volvo define-se como torção de um segmento do tubo digestivo, que, muitas vezes, leva à obstrução intestinal, portanto, a um quadro de abdome agudo obstrutivo $^{(1)} \mathrm{O}$ volvo de ceco, por sua vez, caracteriza-se por torção axial que ocorre envolvendo o ceco, íleo terminal e cólon ascendente. Se não for tratada pode evoluir para isquemia intestinal, necrose ou perfuração ${ }^{(1)}$. A sua incidência mundial é de 2,8 a 7,1 por milhão de pessoas por ano, sendo responsável por $1 \%-1,5 \%$ de todas as obstruções intestinais em adultos e $25 \%-40 \%$ de todos volvos envolvendo o cólon ${ }^{(2)}$. Geralmente, acomete pacientes jovens, mulheres $^{(3)}$. Postula-se que alguns fatores aumentam o risco, como: aderências pós-operatórias, fase final da gravidez, alta ingestão de fibras, íleo adinâmico, constipação crônica, repouso excessivo ${ }^{(2)}$. Além disso, há uma maior propensão para volvo cecal durante os períodos de doenças clínicas concomitantes (cerca de $12 \%-28 \%$ dos pacientes relatados com volvo cecal já estavam hospitalizados momento da diagnóstico) $)^{(2)}$.

As apresentações clínicas podem ser muito variáveis, desde dor abdominal intermitente, auto-limitada, à dor abdominal aguda associada com estrangulamento intestinal e sepse. Tipicamente, os pacientes têm sintomas recorrentes que consistem em dor generalizada ou localizada no quadrante inferior direito abdominal, distensão abdominal, ruídos hidroaéreos aumentados, timpanismo e resolução de dor após a eliminação de 
flatos. Podem ainda apresentar constipação, náusea e vômitos ${ }^{(2)}$. A duração dos sintomas pode variar de horas para dias ${ }^{1}$. Em casos mais graves, onde já existe gangrena, o paciente pode apresentar febre, sinais de irritação peritoneal e hipotensão ${ }^{(3)}$.

O diagnóstico baseia-se, basicamente, no quadro clínico e exames de imagem, pois os exames laboratoriais não são sensíveis nem específicos para o diagnóstico. No entanto, uma leucocitose significativa ou acidose metabólica pode indicar a presença de comprometimento intestinal ${ }^{(1)}$. A radiografia abdominal pode evidenciar alguns sinais sugestivos: dilatação cecal (98\% -100\%), nível único de líquido (72\% -88\%), pequena dilatação do intestino ( $42 \%$-55\%), e ausência de gás no cólon distal (82\%). Estes resultados ajudam a levantar a suspeita de volvo cecal, o que levam a posterior confirmação pelo enema opaco, colonoscopia ou a tomografia computadorizada $(\mathrm{TC})^{(2)}$. Os três sinais mais comuns associados com o volvo cecal aos exames de imagem são: "Grão de café" (ceco dilatado preenchido com ar e fluido), "sinal do bico" (afilamento discreto na altura do ramo eferente da obstrução) e "sinal do giro" (massa com arquitetura interna contendo fios de tecidos moles que rodam, além de atenuação de gordura e vasos mesentéricos ingurgitados ${ }^{(2)}$.

A exploração cirúrgica via laparoscopia ou laparotomia deve ser realizada quando exames de imagem não conseguem estabelecer um diagnóstico ${ }^{(1)} \mathrm{e}$ tambem é utilizada como tratamento padrão ${ }^{(1)}$.

A ocorrência de volvo cecal é incomum, o diagnóstico diferencial entre outras causas de obstrução com base no quadro clínico é difícil, os achados radiológicos são pouco específicos e há pouca familiaridade com esta condição, o que contribui para os atrasos de diagnóstico e tratamento ${ }^{(2)}$.

O objetivo deste relato é conscientizar os médicos com relação a esta doença através da ilustração do caso e da exposição dos aspectos epidemiológicos, clínicos, diagnósticos e terapêuticos. Visamos promover conhecimento acerca de uma condição patológica rara para reduzir os erros de diagnóstico, além de viabilizar detecções precoces que melhoram prognósticos dos doentes.

\section{Relato do caso}

Paciente do sexo feminino, 41 anos, branca, com antecedente de esclerose múltipla com imobilidade crônica, úlcera de pressão na região trocantérica direita e portadora de gastrostomia (GTT) há 2 meses (procedimento realizado por via endoscópica). A mesma foi trazida por familiares ao pronto socorro deste serviço com sinais de necrose em úlcera, evoluindo com sepse grave (rebaixamento do nível de consciência - Glas- gow 8). Após 2 meses de internação, já em tratamento para o quadro infeccioso, iniciou distensão abdominal, vômitos e parada de eliminação de fezes por 3 dias anamnese prejudicada: paciente pouco contactuante devido histórico neurológico. Ao exame se apresentava em regular estado geral, desnutrida grave (IMC: 16), desidratada $(1+/ 4+)$, taquicárdica e normotensa. $\mathrm{O}$ abdome estava distendido, tenso, timpânico, doloroso difusamente a palpação sem defesa, sem visceromegalias, sem sinais de hérnias e com ruídos hidroaéreos presentes. Toque retal com presença de fecaloma, sem massas ou sangramentos. Aos exames laboratoriais, apresentava leucocitose (13700 com desvio até 1\% metamielócitos) e gasometria arterial normal.

Foi realizada radiografia de abdome onde se observou distensão de alças de intestino delgado e ausência de gás em alças colônicas (Imagem 1). Na tomografia de abdome, identificou-se o "sinal do giro", característico de volvo de ceco, na região de fossa ilíaca direita (identificado pela seta vermelha na Imagem 2). Ainda mostrou área de transição abrupta do calibre de alça de intestino delgado na fossa ilíaca direita, determinando importante dilatação entérica à montante, bem como gástrica e esofágica, com formação de nível hidroaéreo.

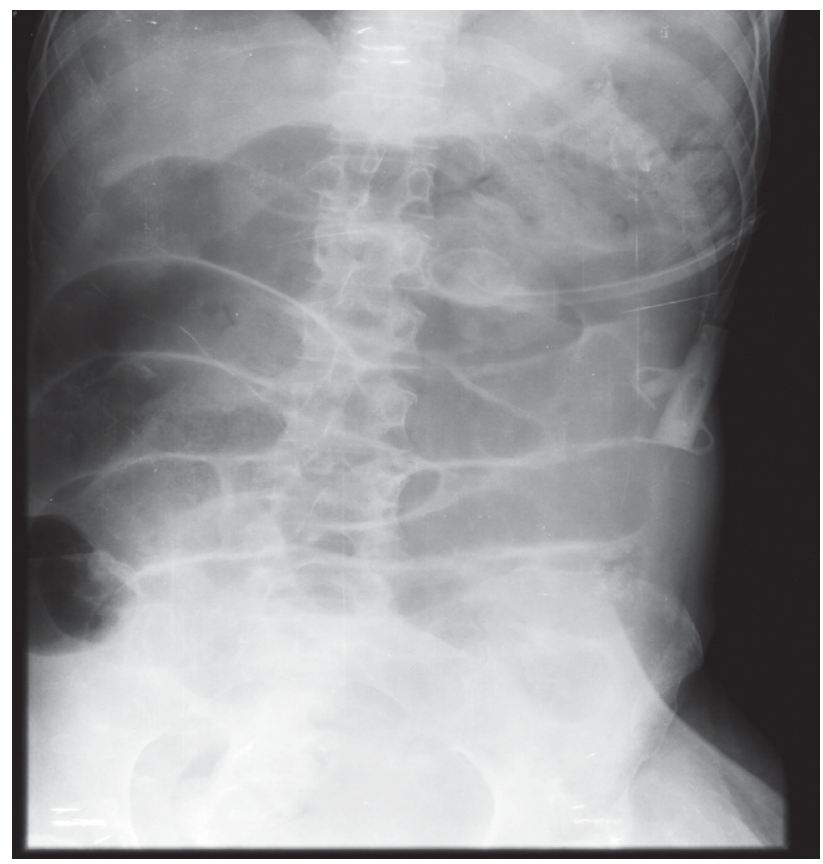

Imagem 1 (imagens cedidas pelo Hospital da Santa Casa de São Paulo)

Na laparotomia exploradora identificou-se distensão de alças, difusamente, de todo intestino delgado e estômago, identificado o volvo de ceco com grande dilatação e localizado em fossa ilíaca direita, porém sem sinais de isquemia importante ou necrose (Imagem 3). Realizada drenagem do conteúdo das alças no 


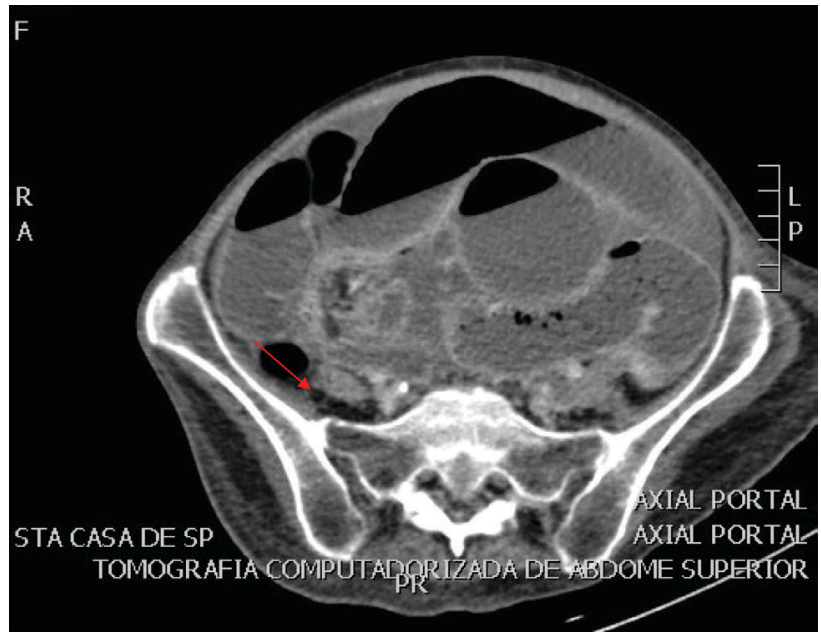

Imagem 2 (imagens cedidas pelo Hospital da Santa Casa de São Paulo)

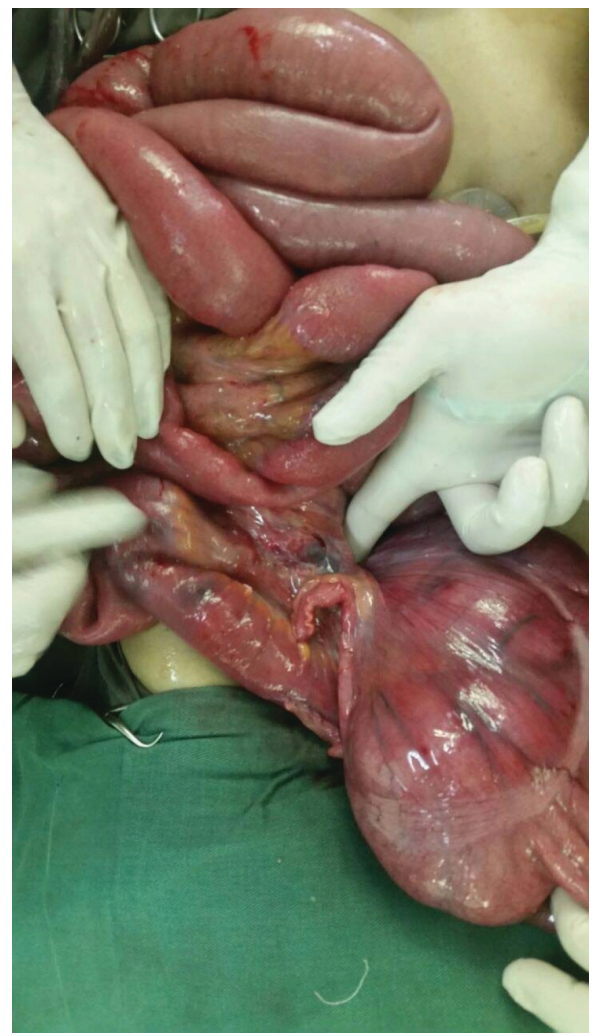

Imagem 3 (imagens cedidas pelo Hospital da Santa Casa de São Paulo)

sentido anti-peristalse; desfeito o volvo, com melhora do aspecto das alças, sem sinais de isquemia. Foi realizada cecopexia na goteira parietocólica direita.

Paciente foi encaminhada ao centro de terapia intensiva, de onde recebeu alta no terceiro dia pós cirurgia. Evoluiu de forma satisfatória, reiniciada dieta enteral pela GTT; ainda mantida NPT, recebeu Polimixina E, Vancomicina e Meropenem, apresentou evacuações e um abdome indolor, com ferida operatória em bom aspecto.

\section{Discussão}

O volvo de ceco pode ser classificado em três tipos $^{(1)}$. O tipo I é chamado volvo cecal axial e se desenvolve a partir da torção axial no sentido horário ou torção do ceco ao longo do seu eixo longo; nestes casos, o ceco permanece no quadrante inferior direito. O tipo II, volvo cecal em laço, desenvolve-se a partir de torção do ceco e de uma porção do íleo terminal; nestes casos, o ceco é transferido para uma localização ectópica (tipicamente quadrante superior esquerdo) e invertido ; a maioria das vezes a torção ocorre em sentido anti-horário. Por fim, o tipo III (báscula cecal) envolve a dobra para cima do ceco em vez de uma torção axial. Os tipos I e II são os mais comuns, correspondendo a cerca de $80 \%$ dos casos, enquanto o tipo III, a $20 \%$ dos $\operatorname{casos}^{(1)}$. No caso apresentado foi visto uma das formas mais comuns, o tipo I.

A epidemiologia da paciente é sugestiva do volvo de ceco, paciente jovem do sexo feminino, com hospitalização atual no momento do diagnóstico ${ }^{(3)}$. O quadro clínico é típico de abdome agudo obstrutivo: dor abdominal, distensão abdominal, parada de eliminação de fezes e vômitos há poucos dias. No exame físico a paciente apresentava abdome distendido, doloroso a palpação difusamente. Uma das características do volvo cecal é distensão assimétrica do abdome, com massa timpânica palpável no quadrante superior esquerdo, o que difere deste caso, que apresentava distensão difusa, o que pode gerar ainda alguma confusão quanto a etiologia $\mathrm{a}^{(4)}$. $\mathrm{O}$ toque retal com fecaloma dificultou o diagnóstico, pois sendo um dos diagnósticos diferenciais para abdome agudo obstrutivo, causou dúvida quanto à etiologia da obstrução ${ }^{(1,4-5)}$.

Para a confirmação do diagnóstico pode-se usar diversos métodos de imagem. O enema de bário demanda mais tempo para realização e apresenta risco de extravasamento de contraste, o que faz com que não seja recomendado para a avaliação de pacientes criticos com obstrução avançada, suspeita de perfuração e intestino gangrenoso. A colonoscopia apresenta baixa taxa de sucesso $(30 \%)$ e há risco de perfuração do cólon, portanto, geralmente, não é recomendada no manejo inicial (2). A TC é a modalidade de imagem preferencial para o diagnóstico (cerca de $90 \%$ dos casos são identificados através da TC). Dessa forma, nesse caso, foi escolhido a TC de abdome que mostrou a presença do "sinal de giro" (torção do mesentério ao redor dos vasos ileocólicos), patognomônico de volvo $^{(1,4,6)}$. Conforme diagnóstico muito sugestivo de volvo de ceco, mostrado em TC, optou-se pelo tratamento cirúrgico: laparotomia exploradora. 
O volvo de ceco, se não tratado rapidamente, pode evoluir com gangrena e perfuração intestinal, sendo assim, deve ser tratado logo após o diagnóstico(2,6). Uma vez que a conduta não operatória de descompressão é raramente possível, cabe ao cirurgião adequar o melhor tratamento ao caso encontrado, tendo como escolha: colonoscopia, cirurgias corretivas ou ressecção da porção acometida ${ }^{(5)}$.

A ressecção do cólon ascendente tem taxa de mortalidade e insulto fisiológico maiores; geralmente é feita em pacientes estáveis. Na cecostomia e cecopexia, a incidência de recorrência de volvo é maior quando comparada à colectomia; usualmente são realizadas em pacientes instáveis ou com complicações. Ancorar o ceco no peritônio parietal reduz o risco de recidiva ${ }^{(3)}$. Os pacientes que têm evidências de comprometimento intestinal (isquemia, necrose ou perfuração) não devem ter o volvo destorcido, devendo então ser submetidos a ressecção do intestino comprometido ${ }^{(1)}$. A escolha da estratégia operatória mais apropriada varia para cada paciente, levando em consideração o estado fisiológico do paciente, a viabilidade dos intestinos envolvidos, o potencial de morbimortalidade perioperatória, o risco de recorrência de volvo e a familiaridade do cirurgião com a técnica ${ }^{(2)}$. Durante o intra-operatório confirmou-se que o paciente apresentava um volvo de ceco tipo I, uma vez que havia torção do ceco em torno de seu próprio eixo, mantendo sua topografia em fossa ilíaca direita ${ }^{(1)}$.

Independente do procedimento de escolhas, todos apresentam, em variados graus, risco de mortalidade, redução do insulto fisiológico, infecção, íleo paralítico e chance de recidiva se não for retirada a porção acometida ${ }^{(2-3)}$. Atualmente, a hemicolectomia à direita apresenta menores riscos e, portanto é a mais utilizada $^{(4)}$. No caso relatado, a paciente apresentava desnutrição grave e choque séptico com necessidade de droga vasoativa para estabilidade hemo-dinâmica durante o intraoperatório, levando a um aumento dos riscos cirúrgicos e da morbimortalidade, por isso foi-se optado realizar a desvolvulação com cecopexia. Esta abordagem geralmente não é a de escolha devido às maiores taxas de recidiva em comparação com a colectomia ${ }^{(3)}$, todavia, devido às condições da paciente, a técnica escolhida se apresentou como opção terapêutica mais rápida e resolutiva no momento. A paciente evoluiu com pós-operatório sem complicações.

\section{Comentários Finais}

O volvo cecal é uma causa rara de obstrução $a b-$ dominal, e uma vez que as manifestações clínicas são inespecíficas, é pouco cogitada em casos de abdome agudo. Este relato traz aspectos epidemiológicos, clínicos e radiológicos que são úteis ao corpo médico no âmbito de diagnóstico mais preciso e rápido, contribuindo com o prognóstico dos pacientes e evitando complicações.

\section{Referências}

1. Maciel LC, Salán FO, Guisard GC. Obstrução intestinal por volvo de ceco: relato de caso. Rev Col Bras Cir. 2009; 36(1):99100 .

2. Consorti ET, Liu TH. Diagnosis and treatment of caecal volvulus. Postgrad Med J 2005; 81(962):772-6.

3. Ruiz-Tovar J, Calero Garcia P, Morales Castiñeiras V, Martinez Molina E. Vólvulo de ciego: presentación de 18 casos y revisión de la literatura. Cir Esp. 2009; 85 (2):110-3.

4. Fry RD, Mahmoud NN, Maron DJ, Bleier JIS. Cólon e reto. In: Townsend CM Jr, Beauchamp RD, Evers BM, Mattox KL. Sabiston tratado de cirurgia: a base biológica da biológica da prática cirúrgica moderna. 19. a ed. Rio de Janeiro: Elsevier; 2015. v. 2, p.1314-7.

5. Destro MWB, Destro C, Salles VEJ, Cauduro AB, Kalume RS. Volvo de ceco no pós-operatório recente de abdominoplastia. Rev Soc Bras Cir Plast. 2007; 22(3): 176-9.

6. Rosenblat JM, Alla BA, Rozenblit M, Wolf EL, DuBrow RA, Den EI, et al. Findings of cecal volvulus at CT. Radiology. 2010; 256 (1):169-75.

Trabalho recebido: $21 / 11 / 2016$

Trabalho aprovado: 18/10/2017 